\title{
Neuroprotective effects of Gymnema sylvestre on streptozotocin-induced diabetic neuropathy in rats
}

\author{
AMAL JAMIL FATANI ${ }^{1}$, SALIM SALIH AL-REJAIE ${ }^{1}$, HATEM MUSTAFA ABUOHASHISH ${ }^{1,2}$, \\ ABDULLAH AL-ASSAF ${ }^{3}$, MIHIR YOGESHKUMAR PARMAR ${ }^{1}$, MOHAMMAD SHAMSUL OLA ${ }^{4}$ \\ and MOHAMMED MAHBOOBUDDIN AHMED ${ }^{1}$
}

\author{
${ }^{1}$ Department of Pharmacology and Toxicology, College of Pharmacy, King Saud University, Riyadh 11544; \\ ${ }^{2}$ Department of Biomedical Dental Sciences, College of Dentistry, University of Dammam, Dammam, Eastern \\ Province 31441; ${ }^{3}$ Department of Food Science and Nutrition, College of Food and Agricultural Sciences; \\ ${ }^{4}$ Department of Biochemistry, College of Science, King Saud University, Riyadh 11544, Saudi Arabia
}

Received December 22, 2014; Accepted February 10, 2015

DOI: $10.3892 /$ etm.2015.2305

\begin{abstract}
The application of traditional medicine for diabetes and associated complications, such as diabetic neuropathy (DN), has received increasing attention. The aim of the present study was to investigate the potential ameliorative effect of Gymnema sylvestre (Gs) in a rat model of DN. Diabetes was induced via a single intraperitoneal injection of streptozotocin (STZ; $60 \mathrm{mg} / \mathrm{kg}$ ). Treatment with Gs extract (50 or $100 \mathrm{mg} / \mathrm{kg} /$ day) began two weeks following the administration of STZ and was continued for five weeks. Pain threshold behavior tests were performed subsequent to the five-week Gs treatment period. In addition, the serum levels of glucose, insulin and proinflammatory cytokines, including tumor necrosis factor (TNF)- $\alpha$, interleukin (IL)- $1 \beta$ and IL-6, were determined. Furthermore, the sciatic tissue levels of nitric oxide, thiobarbituric acid reactive substances and reduced glutathione were determined, as well as the activity levels of superoxide dismutase, catalase, glutathione peroxidase and glutathione reductase. Levels of insulin-like growth factor (IGF), nerve growth factor (NGF), TNF- $\alpha$, IL-1 $\beta$ and IL-6 were also assessed in the sciatic tissue. In addition, the sciatic nerve tissue samples were analyzed for histopathological alterations. The diabetic rats exhibited apparent reductions in the paw-withdrawal $(31 \%$; $\mathrm{P}<0.01)$ and tail-flick latencies (38\%; $\mathrm{P}<0.05)$. Furthermore, the diabetic rats demonstrated an evident elevation in serum and sciatic levels of proinflammatory cytokines. Measured oxidative stress biomarkers were significantly altered in the sciatic nerve tissue of the diabetic rats. Treatment with Gs attenuated diabetes-induced modifica-
\end{abstract}

Correspondence to: Professor Salim Salih Al-Rejaie, Department of Pharmacology and Toxicology, College of Pharmacy, King Saud University, 21 Abdullah Road, Riyadh 11544, Saudi Arabia E-mail: rejaie@ksu.edu.sa

Key words: Gymnema sylvestre, hyperglycemic neuropathy, inflammation, oxidative stress tions with regard to the levels of serum glucose, insulin and proinflammatory cytokines. In the sciatic nerve tissue, the diabetes-induced alterations in IL levels and oxidative stress biomarkers were significantly improved in the Gs-treated rats. Furthermore, the reduction in the sciatic tissue expression levels of IGF and NGF was also ameliorated by Gs treatment. Histological analysis indicated that Gs corrected the sciatic tissue in the diabetic rats. Therefore, the results demonstrated that the neuroprotective effect of Gs may be associated with the inhibitory effect on the excessive activation of inflammatory molecules and oxidative stress mediators.

\section{Introduction}

Diabetic neuropathy (DN) is one of the major complications associated with diabetes. A previous clinical study indicated that DN affects $15-25 \%$ of type 1 and $30-40 \%$ of type 2 diabetic patients (1). The symptoms of DN include burning sensations, hyperalgesia, allodynia and dysesthesia, which may substantially impact the patient quality of life and emotional well-being (2). A number of disorders have been associated with DN, including vascular (3), metabolic (4) and neurotrophic abnormalities (5). These dysfunctions may lead to electrophysiological alterations, abnormal sensory perception and nerve fiber damage (6). Furthermore, DN is characterized by neuronal degeneration and modifications in the activity of nerve growth factor (NGF) and insulin-like growth factor (IGF) (7).

Oxidative stress has been suggested as a potential pathophysiological mechanism underlying $\mathrm{DN}$ and its involvement in the development of other diabetic complications (8). Sciatic nerve dysfunction and reduced endoneurial blood flow have been observed in diabetic rats following exposure to oxidative stress $(9,10)$. Furthermore, hyperglycemia has been demonstrated to activate lipid peroxidation (LPO) and induce the overproduction of reactive oxygen species (ROS) in the sciatic nerve (11). Thus, diabetes-induced oxidative stress may also damage the sciatic nerve similar to damage induced by oxidative stress in other tissues.

Gymnema sylvestre (Gs), from the Asclepiadaceae family, has been used as a traditional medicine in southern India, 
tropical Africa and Australia for almost 2,000 years $(12,13)$. Previous studies have indicated that Gs may possess certain benefits in improving urination, stomach ulceration and diabetes (14-16). The hyperglycemia-induced elevation in urea, uric acid and creatinine levels and the associated decline in the glomerular filtration rate were significantly inhibited following the Gs treatment in diabetic rats (15). Furthermore, previous studies have indicated that the leaves of Gs contain gymnemic acids, which are a group of triterpenoid saponins $(13,17)$, alkaloids, acidic glycosides and anthroquinones (18). Prior studies demonstrated that an extract from Gs leaves exhibited antioxidative and LPO-inhibiting activity in an experimental model of gastric ulceration and colitis $(19,20)$. As the pathogenesis of DN is characterized by increased levels of ROS and an associated inflammatory response, we hypothesized that the antioxidative effect of Gs may function as an anti-inflammatory therapy to ameliorate nerve tissue damage. Therefore, in the present study, the antioxidative and anti-inflammatory potential of Gs leaf extract was investigated in an streptozotocin (STZ)-induced model of DN in the sciatic nerves of diabetic male Wistar rats.

\section{Materials and methods}

Animals. A population of 24 male, 8-week old Wistar albino rats, weighing 270-290 g, was obtained from the Experimental Rat Care Center of the College of Pharmacy at King Saud University (Riyadh, Saudi Arabia). The rats were housed under controlled conditions $\left(25^{\circ} \mathrm{C}\right.$ and a 12 -h light/dark cycle) and had free access to water and Purina rat chow (Grain Silos and Flour Mills Organization, Riyadh, Saudi Arabia). All experimental procedures and protocols, including anesthesia, were in accordance with the National Institutes of Health (NIH) Guide for the Care and Use of Laboratory Animals, Institute for Laboratory Animal Research (NIH Publications no. 80-23, 1996). In addition, ethical approval (no. 235-EACC-2014) was granted by the Ethical Committee of the Experimental Animal Care Center of the College of Pharmacy at King Saud University.

Plant extract. 'Diaglu' capsules containing an ethanol extract of dried Gs leaves were purchased from Mepaco-Medifood (Cairo, Egypt). The extract was standardized with $25 \%$ gymneric acids as the major constituent, in addition to anthroquinones and their derivatives as described by the manufacturer. In a previous study, a phytochemical analysis was conducted in our laboratory (19) and approximately the same amount of gymneric acids were detected in the extract. The dried powder was suspended in $0.25 \%$ carboxymethyl cellulose solution (Loba Chemie Pvt., Ltd., Mumbai, India) and administered orally by gavage in either a low dose $(50 \mathrm{mg} / \mathrm{kg} / \mathrm{day})$ or high dose $(100 \mathrm{mg} / \mathrm{kg} /$ day $)$. In a previous study, a phytochemical analysis of Gs dried ethanolic leaves extract was conducted and the phytochemical constituents present in the extract were reported (19).

Diabetes induction. Diabetes was chemically induced by an intraperitoneal injection of freshly prepared STZ (60 mg/kg; Sigma-Aldrich, St. Louis, MO, USA) in $0.1 \mathrm{~mol} / 1$ citrate-buffered solution ( $\mathrm{pH} 4.5$ ). A group of vehicle control rats were injected with an equal volume of $0.1 \mathrm{~mol} / 1$ citrate buffer. Four days following the STZ injection, diabetes induction was confirmed by measuring the fasting blood glucose levels in a tail vein blood sample using an Accu-Chek Compact Plus glucose meter system (Roche Diagnostics, Meylan, France). Rats with a glucose level of $\geq 250 \mathrm{mg} / \mathrm{dl}$ were classified as diabetic.

Experimental design. Animals were divided into normal control and diabetic rats and further allocated into four groups ( $n=6$ per group), which included the control, diabetic, diabetic rats treated with $50 \mathrm{mg} / \mathrm{kg} /$ day Gs (GS50 group) and diabetic rats treated with $100 \mathrm{mg} / \mathrm{kg} /$ day Gs (GS100 group). Doses of 50 and $100 \mathrm{mg} / \mathrm{kg} /$ day were selected for this experiment based on previous studies $(19,20)$. Treatment was initially administered orally by gavage two weeks following the STZ injection, and was continued for five consecutive weeks. Subsequent to the treatment, the rats were fasted overnight then blood samples were collected via cardiac puncture, under anesthetic (diethyl ether; Sigma-Aldrich). The blood samples were centrifuged at $1800 \mathrm{x} \mathrm{g}$ for $10 \mathrm{~min}$ at room temperature (Hettich EBA-20; Andreas Hettich GmbH \& Co. KG, Tuttlingen, Germany) and the serum was collected and stored at $-20^{\circ} \mathrm{C}$ until required for further analysis. The sciatic nerves were dissected immediately and a small cross section from each group was fixed in $10 \%$ formaldehyde solution for histopathological evaluation. The remaining sciatic nerve tissues were stored at $-75^{\circ} \mathrm{C}$ for biochemical analysis.

Mechanical hyperalgesia (Randall-Selitto method). The mean right and left paw pressure thresholds were determined using an MK-20D paw pressure analgesia meter (Muromachi Kikai Co. Ltd., Tokyo, Japan). The pressure linear increase rate was fixed at $10 \mathrm{mmHg} / \mathrm{sec}$ and the cut-off pressure was set at $500 \mathrm{mmHg}$ in order to avoid tissue injury. Pressure was applied to the center of the hind paw, and when the rats displayed pain by withdrawal of the paw, the applied paw pressure was recorded by the analgesia meter and expressed in $\mathrm{mmHg}$. Three tests were performed for each rat, separated by at least $10 \mathrm{~min}$, and the mean value was recorded.

Tail flick test. Acute nociception was induced using a Socrel model DS 20 tail flick apparatus (Apelex, Bagneux, France). In brief, the tail flick latency of the restrained rats was measured by focusing an intensity-controlled beam of light on the distal $2 \mathrm{~cm}$ of the rat tail. Next, the period required for the rat to remove the tail from this thermal stimulus was recorded. In order to avoid manual error, two or three recordings were measured at an interval of $15 \mathrm{~min}$ for each rat, and the mean value was calculated for statistical analysis.

Serum analysis. Serum levels of glucose and insulin were determined using commercially available diagnostic kits (Randox Laboratories Ltd., Crumlin, County Antrim, UK) and the levels of TNF- $\alpha$, IL-1 $\beta$ and IL- 6 were estimated using enzyme-linked immunosorbent assay (ELISA) kits (R\&D Systems, Inc., Minneapolis, MN, USA).

Estimation of the levels of thiobarbituric acid reactive substances (TBARS) in the sciatic nerve. Levels of the LPO 
product, malondialdehyde (MDA), were estimated using an assay kit for TBARS (ZeptoMetrix Corporation, Buffalo, NY, USA) in the sciatic nerve tissues. In brief, $100 \mu$ l tissue homogenate was mixed with $2.5 \mathrm{ml}$ reaction buffer and heated for $1 \mathrm{~h}$ at $95^{\circ} \mathrm{C}$. Following cooling, the absorbance of the supernatant was measured at $532 \mathrm{~nm}$ by a Pharmacia-LKB UVM II spectrophotometer (GE Healthcare Life Sciences, Marlborough, MA, USA). The LPO products were expressed in terms of nmol MDA/mg protein.

Estimation of reduced glutathione (GSH) levels in the sciatic nerve. GSH levels in the sciatic nerve were determined using the procedure previously described by Sedlak and Lindsay (21). Briefly, $0.5 \mathrm{ml}$ cold EDTA tissue homogenate (0.02 mol/l) was added to $0.2 \mathrm{~mol} / 1$ Tris buffer $(\mathrm{pH} \mathrm{8.2)}$ and $0.1 \mathrm{ml} \mathrm{5,5}$-dithiobis-(2-nitrobenzoic acid) $(0.01 \mathrm{~mol} / \mathrm{l}$; $\mathrm{BDH}$ Chemicals Ltd., Poole, England). Samples were centrifuged at $1350 \mathrm{x} \mathrm{g}$ for $10 \mathrm{~min}$ at room temperature (Hettich EBA-20). The absorbance of the clear supernatant was measured at $412 \mathrm{~nm}$ using a Pharmacia-LKB UVM II spectrophotometer (GE Healthcare Life Sciences).

Estimation of the activity levels of superoxide dismutase (SOD), catalase (CAT), glutathione peroxidase (GPX) and glutathione reductase $(G R)$ in the sciatic nerve. Enzymatic activity levels of SOD in the post-mitochondrial supernatant of the sciatic nerve homogenate were measured following the method described by Kono (22). Superoxide anions generate hydroxylamine hydrochloride by oxidation, mediating the reduction of nitroblue tetrazolium to form blue formazan, which was subsequently measured at $560 \mathrm{~nm}$ under aerobic conditions. SOD inhibits the nitroblue tetrazolium reduction, and the extent of this inhibition was used as a measure of SOD activity and expressed as U/mg protein. The CAT enzymatic activity was measured according to the method described by Aebi et al (23). In brief, the post-mitochondrial supernatant of the sciatic nerve homogenate was mixed with $50 \mathrm{mmol} / \mathrm{l}$ phosphate buffer ( $\mathrm{pH} 7.0$ ) and $20 \mathrm{mmol} / \mathrm{l}_{2} \mathrm{O}_{2}$. The enzymatic activity of CAT was subsequently determined based on the reduction in absorbance at $240 \mathrm{~nm}$ and expressed in $\mathrm{U} / \mathrm{mg}$ protein. The enzymatic activities of GR and GPx were determined using commercially available colorimetric kits (WuXi PharmaTech Cayman, Inc., New York, NY, USA).

Estimation of the levels of $I L-1 \beta, I L-6, T N F-\alpha, N O, N G F$ and IGF in the sciatic nerve. Levels of IL-1 $\beta$, IL- 6, TNF- $\alpha$, NGF and IGF in the sciatic nerve were determined using ELISA kits (R\&D Systems, Inc.), and the results were recorded as pg/mg protein. The NO concentrations in the sciatic nerve tissues were estimated via the Griess test, using a commercially available kit (R\&D Systems, Inc.).

Western blot analysis. In order to determine the NGF protein expression levels, the sciatic nerve tissues were homogenized in a buffer containing $10 \mathrm{mM}$ HEPES (pH 7.4), $100 \mathrm{mM} \mathrm{NaCl}$, $1 \mathrm{mM} \mathrm{Na} \mathrm{VO}_{4}, 10 \mathrm{mM}$ sodium pyrophosphate, $10 \mathrm{mM} \mathrm{NaF}$, 2 mM EDTA, 1 mM PMSF, 1\% Triton X-100, 0.2\% SDS and a protease inhibitor cocktail. The samples were centrifuged at $15,000 \mathrm{x} \mathrm{g}$ for $10 \mathrm{~min}$, the supernatants were collected and the protein concentrations were estimated using a protein assay kit (Bio-Rad Laboratories, Inc., Hercules, CA, USA). Protein samples were boiled in Laemmli's sample buffer (Bio-Rad Laboratories, Inc.) for $5 \mathrm{~min}$, after which $50 \mu \mathrm{g}$ protein was separated by $10 \%$ SDS-polyacrylamide gel electrophoresis and transferred onto nitrocellulose membranes (Bio-Rad Laboratories, Inc.). The membranes were blocked for $90 \mathrm{~min}$ at room temperature with 5\% non-fat milk in Tris-buffered saline containing $0.1 \%$ Tween-20 (TBS-T). Next, the membranes were incubated overnight with a rabbit anti-NGF antibody $(1 \mu \mathrm{g} / \mathrm{ml}$; \#Ab6199, Abcam, Cambridge, UK). Following overnight incubation with the primary antibody, the membranes were washed three times with TBS-T (5 min each) and incubated with a respective secondary horseradish peroxidase-conjugated antibody (1:2,000; Santa Cruz Biotechnology, Inc., Santa Cruz, CA, USA) for $90 \mathrm{~min}$ at room temperature. Subsequently, the membranes were washed four times with TBS-T ( 5 min each) and the immunoreactivity of the bands was visualized on a C-DiGit blot scanner (LI-COR Biosciences, Lincoln, NE, USA) using enhanced chemiluminescence with a western blotting luminol reagent (1:1; Santa Cruz Biotechnology, Inc.). Protein bands were quantified by densitometric analysis using Image-Lab software, version 2.0.1 (Bio-Rad Laboratories, Inc.). For an internal control, the membranes were washed and incubated with a monoclonal mouse $\beta$-actin antibody $(1: 2,000$; Santa Cruz Biotechnology, Inc.), according to the aforementioned method.

Histopathological assessment of the sciatic nerve. Cross sections of the sciatic nerve tissue were fixed in $10 \%$ formaldehyde solution, embedded into paraffin wax blocks and cut into $5 \mu \mathrm{m}$ slices using a microtome (American Optical Rotary Mirotome, Middleton, WI, USA). The samples were stained with hematoxylin and eosin, mounted and observed microscopically by a histopathologist in a blinded fashion using a Leica DM5500 B (Leica Biosystems Melbourne Pty Ltd., Melbourne, Australia). A microscopic description for all the samples was graded accordingly as mild, moderate or severe neuropathy for the sciatic nerves.

Statistical analysis. Results are expressed as the mean \pm standard error of the mean, and statistical analysis was conducted using one-way analysis of variance followed by the Student-Newman-Keuls post hoc test. $\mathrm{P} \leq 0.05$ was considered to indicate a statistically significant difference. All statistical analyses were conducted using GraphPad Prism 5 and Instat statistical software (GraphPad Software, Inc., La Jolla, CA, USA).

\section{Results}

Effects of Gs on mechanical and thermal pain threshold in diabetic rats. The paw pressure analgesia and tail flick tests indicated a significant reduction in the pain threshold of the diabetic rats when compared with the control rats. Administration of the higher dose of Gs (100 mg/kg/day) to the diabetic rats for five consecutive weeks resulted in a significant $(\mathrm{P}<0.05)$ improvement in the tail withdrawal latency time (Fig. 1).

Effects of Gs on body weight, blood glucose and insulin levels in diabetic rats. The mean body weight of the diabetic rats was significantly $(\mathrm{P}<0.001)$ reduced and this symptom was 
A

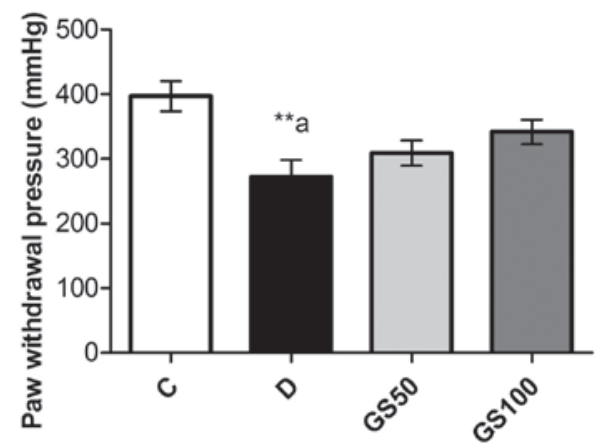

B

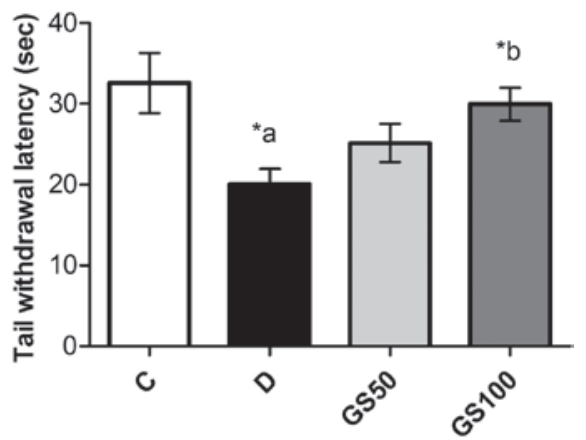

Figure 1. Effects of Gs on (A) mechanical and (B) thermal analgesia in diabetic rats. Data are expressed as the mean \pm standard error of the mean ( $\mathrm{n}=6$ ) and were analyzed using one-way analysis of variance followed by the Student-Newman-Keuls post hoc test. ${ }^{* a} \mathrm{P}<0.05$ and ${ }^{* * a} \mathrm{P}<0.01$, vs. C group; ${ }^{* b} \mathrm{P}<0.05$, vs. D group. C, control group; D, diabetic group; GS50, rats received $50 \mathrm{mg} / \mathrm{kg} /$ day Gs extract; GS100, rats received $100 \mathrm{mg} / \mathrm{kg} / \mathrm{day}$ Gs extract; Gs, Gymnema sylvestre.
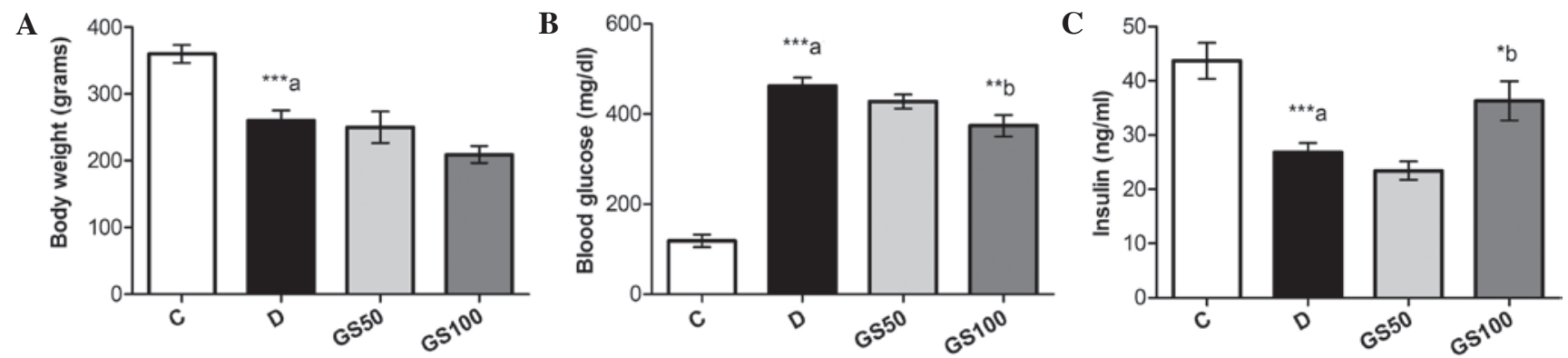

Figure 2. Effects of Gs leaf extract on (A) body weight, and the serum levels of (B) glucose and (C) insulin in diabetic rats. Data are expressed as the mean \pm standard error of the mean $(n=6)$ and were analyzed using one-way analysis of variance followed by the Student-Newman-Keuls post hoc test. ${ }^{* * * a} \mathrm{P}<0.001$, vs. C group; ${ }^{*} \mathrm{P}<0.05$ and ${ }^{* *} \mathrm{P}<0.01$, vs. D group. C, control group; D, diabetic group; GS50, rats received $50 \mathrm{mg} / \mathrm{kg} / \mathrm{day}$ Gs extract; GS100, rats received $100 \mathrm{mg} / \mathrm{kg} /$ day Gs extract; Gs, Gymnema sylvestre.
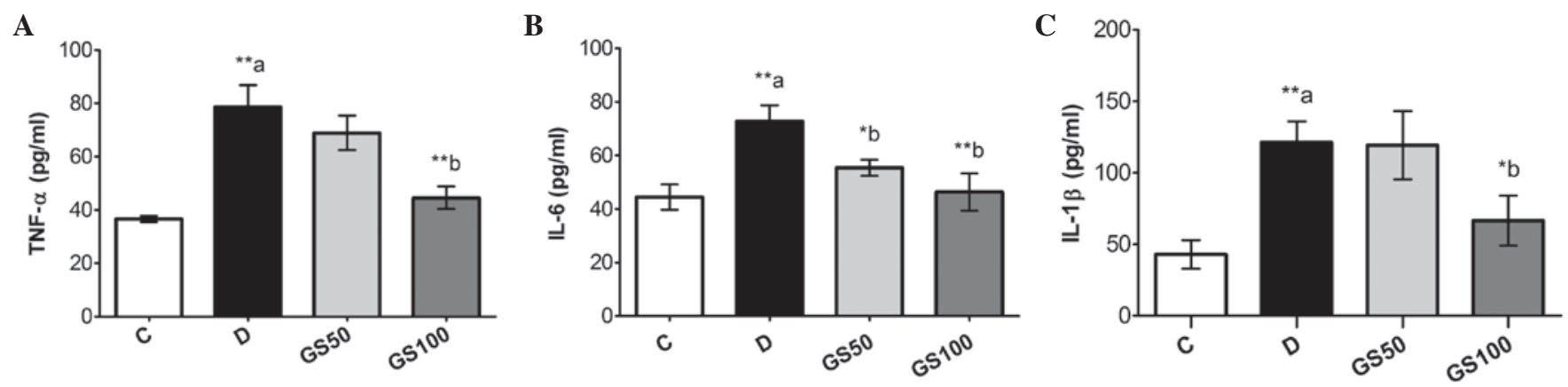

Figure 3. Effects of Gs leaf extract on the levels of (A) TNF- $\alpha$, (B) IL-6 and (C) IL-1 $\beta$ in the serum of diabetic rats. Data are expressed as the mean \pm standard error of the mean $(n=6)$ and were analyzed using one-way analysis of variance followed by the Student-Newman-Keuls post hoc test. ${ }^{* * a} \mathrm{P}<0.01$, vs. $\mathrm{C}$ group; ${ }^{* b} \mathrm{P}<0.05$ and ${ }^{* * \mathrm{~b}} \mathrm{P}<0.01$, vs. D group. C, control group; D, diabetic group; GS50, rats received $50 \mathrm{mg} / \mathrm{kg} / \mathrm{day} \mathrm{Gs}$ extract; GS100, rats received 100 mg/kg/day Gs extract; TNF, tumor necrosis factor; IL, interleukin; Gs, Gymnema sylvestre.

not ameliorated following treatment with Gs. However, the increased blood glucose levels present in the diabetic rats were significantly $(\mathrm{P}<0.01)$ mitigated by treatment with the higher dose of Gs (100 mg/kg/day). Furthermore, while insulin levels were markedly $(\mathrm{P}<0.001)$ reduced in the diabetic rats, a significant $(\mathrm{P}<0.05)$ increase was observed in the levels of insulin in the GS100 group rats when compared with the untreated diabetic rats (Fig. 2).

Effects of Gs on levels of proinflammatory cytokines in diabetic rats. Serum levels of proinflammatory cytokines, including TNF- $\alpha$, IL-1 $\beta$ and IL-6, were significantly $(\mathrm{P}<0.01)$ increased in the diabetic rats, as compared with the control group rats. These inflammatory mediators were markedly inhibited in the GS100 group rats when compared with the untreated diabetic rats. However, treatment with the lower dose of $\mathrm{Gs}(50 \mathrm{mg} / \mathrm{kg} /$ day $)$ resulted in the significant $(\mathrm{P}<0.05)$ inhibition of IL-6 levels only (Fig. 3).

Significant $(\mathrm{P}<0.01)$ elevations were observed in the levels of inflammatory biomarkers, including TNF- $\alpha$, IL-1 $\beta$, IL- 6 and $\mathrm{NO}$, in the sciatic nerves of the diabetic rats. However, the GS100 group rats exhibited significantly attenuated elevated levels of NO, IL-1 $\beta(\mathrm{P}<0.05)$, IL-6 and TNF- $\alpha(\mathrm{P}<0.01)$ in the sciatic nerves when compared with the untreated diabetic rats (Fig. 4). 

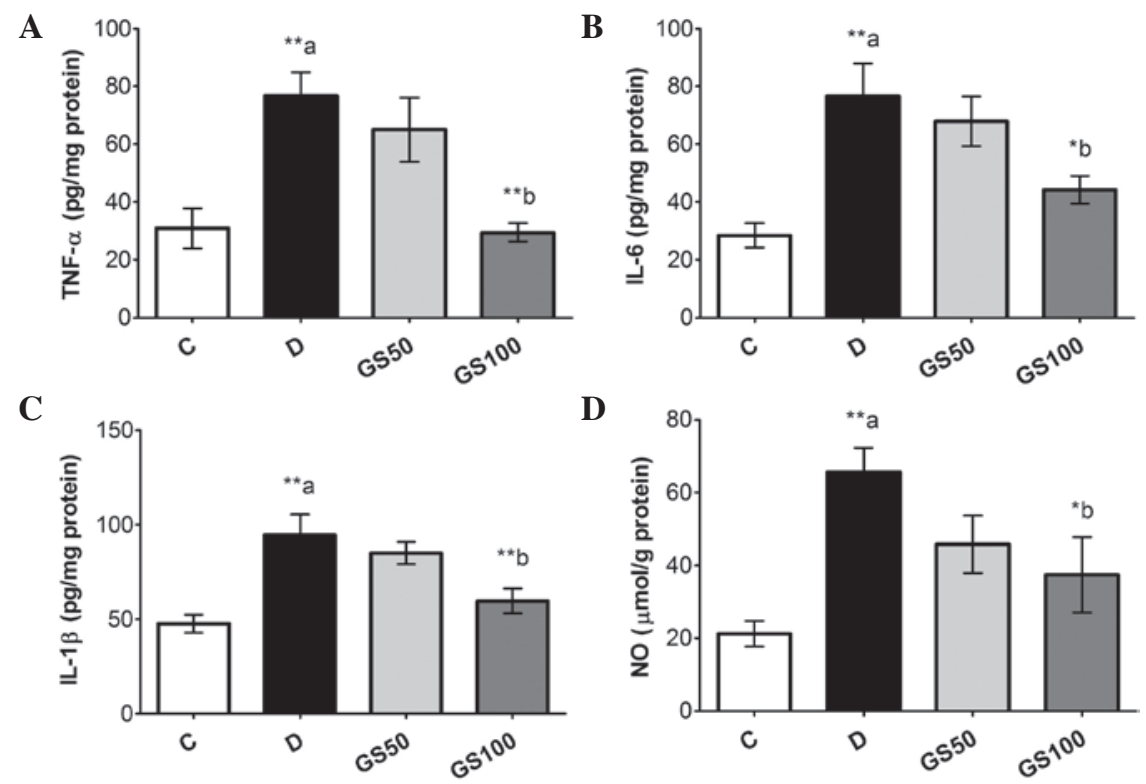

Figure 4. Effects of Gs leaf extract on the levels of (A) TNF- $\alpha$, (B) IL-6, (C) IL-1 $\beta$ and (D) NO in the sciatic nerves of diabetic rats. Data are expressed as the mean \pm standard error of the mean $(n=6)$ and were analyzed using one-way analysis of variance followed by the Student-Newman-Keuls post hoc test. ${ }^{* * a} \mathrm{P}<0.01$, vs. C group; ${ }^{* b} \mathrm{P}<0.05$ and ${ }^{* * b} \mathrm{P}<0.01$, vs. D group. C, control group; D, diabetic group; GS50, rats received 50 mg/kg/day Gs extract; GS100, rats received $100 \mathrm{mg} / \mathrm{kg} / \mathrm{day}$ Gs extract; TNF, tumor necrosis factor; IL, interleukin; NO, nitric oxide; Gs, Gymnema sylvestre.

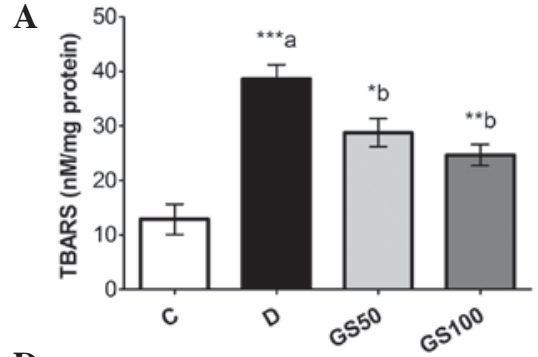

D

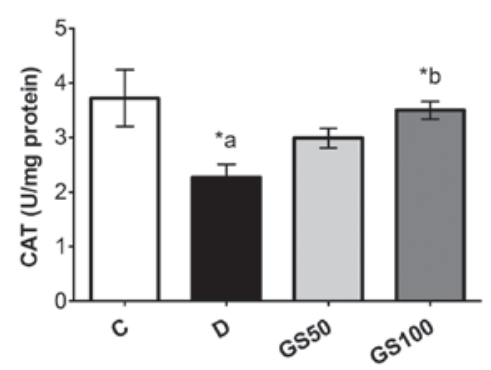

B

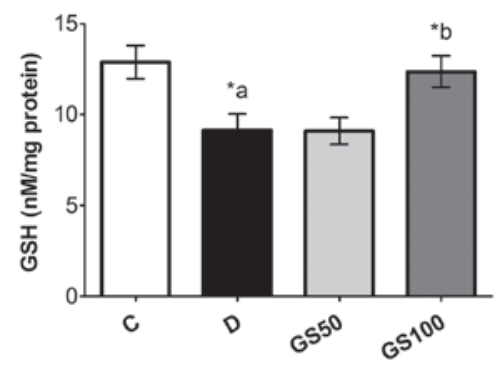

$\mathbf{E}$

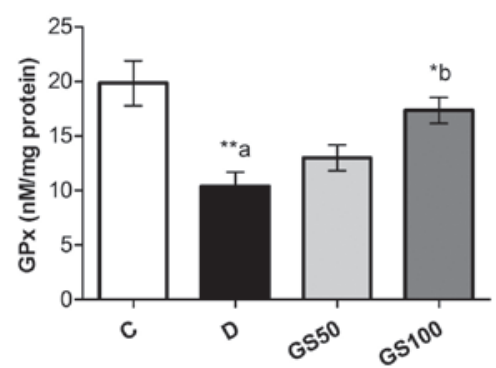

C

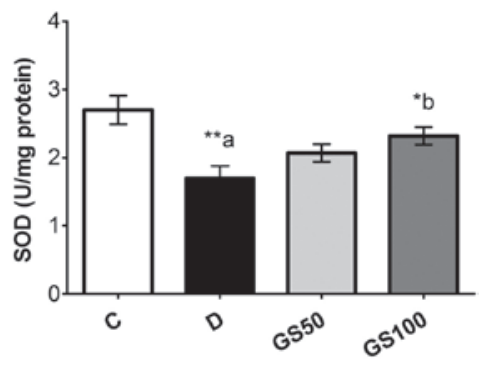

$\mathbf{F}$

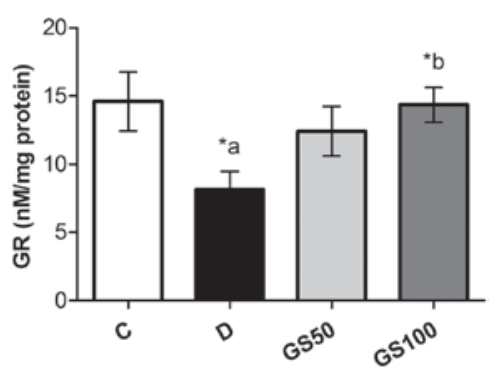

Figure 5. Effects of Gs leaf extract on the levels of (A) TBARS and (B) GSH, and the enzymatic activity levels of (C) SOD, (D) CAT, (E) GPx and (F) GR in the sciatic nerves of diabetic rats. Data are expressed as the mean \pm standard error of the mean $(n=6)$ and were analyzed using one-way analysis of variance followed by the Student-Newman-Keuls post hoc test. ${ }^{* a} \mathrm{P}<0.05,{ }^{* *} \mathrm{P}<0.01$ and ${ }^{* * * *} \mathrm{P}<0.001$, vs. C group; ${ }^{*} \mathrm{P}<0.05$ and ${ }^{* *} \mathrm{P}<0.01$, vs. D group. C, control group; $\mathrm{D}$, diabetic group; GS50, rats received $50 \mathrm{mg} / \mathrm{kg} /$ day Gs extract; GS100, rats received $100 \mathrm{mg} / \mathrm{kg} /$ day Gs extract; TBARS, thiobarbituric acid reactive substance; GSH, glutathione; SOD, superoxide dismutase; CAT, catalase; GPx, glutathione peroxidase; GR, glutathione reductase; Gs, Gymnema sylvestre.

Effects of Gs on LPO and oxidative stress bio-markers in diabetic rats. The induction of diabetes in the rats resulted in significantly $(\mathrm{P}<0.001)$ elevated levels of TBARS in the sciatic nerve tissue. Furthermore, the diabetic rats exhibited reduced levels of GSH $(\mathrm{P}<0.05)$ and lower enzymatic activity levels of SOD $(\mathrm{P}<0.01)$, CAT $(\mathrm{P}<0.05)$, GPx $(\mathrm{P}<0.01)$ and GR $(\mathrm{P}<0.05)$ when compared with the control rats. The GS50 and GS100 group rats exhibited significant inhibition of the diabetes-induced elevation of TBARS in the sciatic nerve, at $\mathrm{P}<0.05$ and $\mathrm{P}<0.01$, respectively. However, enhanced levels of GSH $(\mathrm{P}<0.05)$, as compared with the untreated diabetic rats, were observed only in the GS100 group rats. Furthermore, the inhibited antioxidant enzymatic activity levels of SOD, CAT, GPx and GR in the sciatic nerve were markedly improved $(\mathrm{P}<0.05)$ in the GS100 group rats (Fig. 5).

Effects of Gs on sciatic levels of IGF and NGF in diabetic rats. Sciatic levels of IGF and NGF were significantly $(\mathrm{P}<0.05$ and $\mathrm{P}<0.01$; respectively) reduced in the diabetic rats when compared with the control rats. However, the GS100 group rats exhibited significant $(\mathrm{P}<0.05)$ reductions in the levels of IGF and NGF when compared with the untreated diabetic rats (Fig. 6). 
A

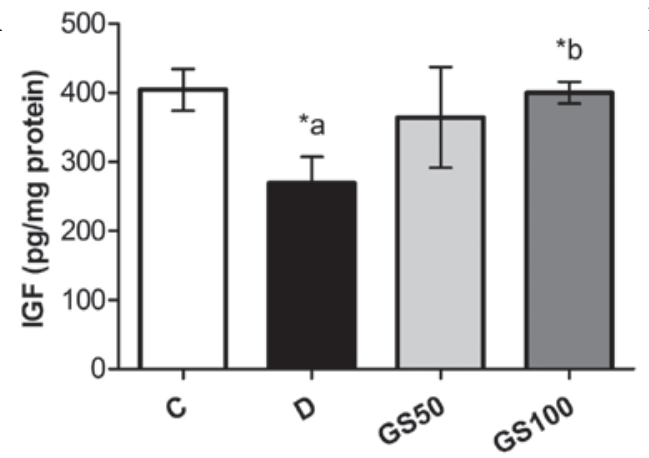

B

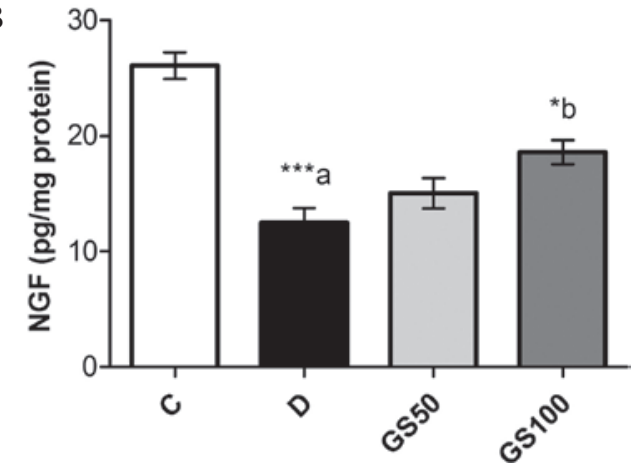

Figure 6. Effects of Gs leaf extract on the levels of (A) IGF and (B) NGF in the sciatic nerves of diabetic rats. Data are expressed as the mean \pm standard error of the mean $(\mathrm{n}=6)$ and were analyzed using one-way analysis of variance followed by the Student-Newman-Keuls post hoc test. ${ }^{* a} \mathrm{P}<0.05$ and ${ }^{* * * a} \mathrm{P}<0.001$, vs. C group; ${ }^{*} \mathrm{P}<0.05$, vs. D group. C, control group; D, diabetic group; GS50, rats received $50 \mathrm{mg} / \mathrm{kg} / \mathrm{day}$ Gs extract; GS100, rats received $100 \mathrm{mg} / \mathrm{kg} / \mathrm{day}$ Gs extract; IGF, insulin-like growth factor; NGF, nerve growth factor; Gs, Gymnema sylvestre.
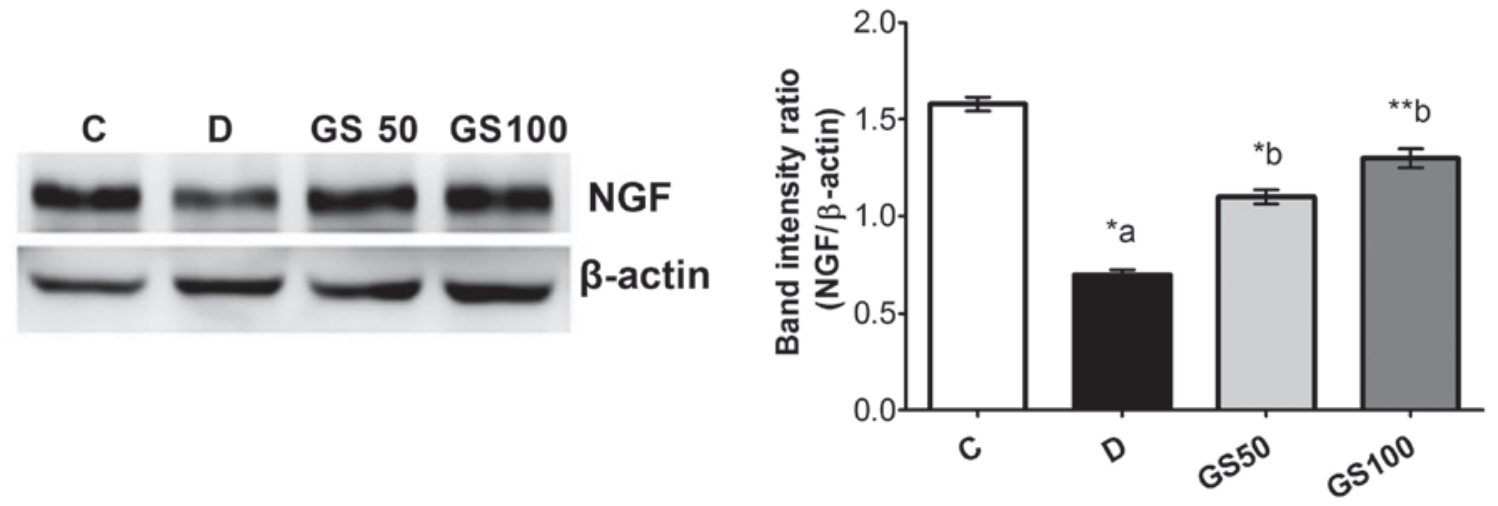

Figure 7. Effects of Gs leaf extract on the protein expression levels of NGF in the sciatic nerves of diabetic rats. Data are expressed as the mean \pm standard error of the mean $(\mathrm{n}=6)$ and were analyzed using one-way analysis of variance followed by the Student-Newman-Keuls post hoc test. ${ }^{*} \mathrm{P}<0.05$, vs. C group; ${ }^{*} \mathrm{P}<0.05$ and ${ }^{* *} \mathrm{P}<0.01$, vs. D group. C, control group; D, diabetic group; GS50, rats received $50 \mathrm{mg} / \mathrm{kg} / \mathrm{day}$ Gs extract; GS100, rats received $100 \mathrm{mg} / \mathrm{kg} / \mathrm{day} \mathrm{Gs} \mathrm{extract}$; NGF, nerve growth factor; Gs, Gymnema sylvestre.
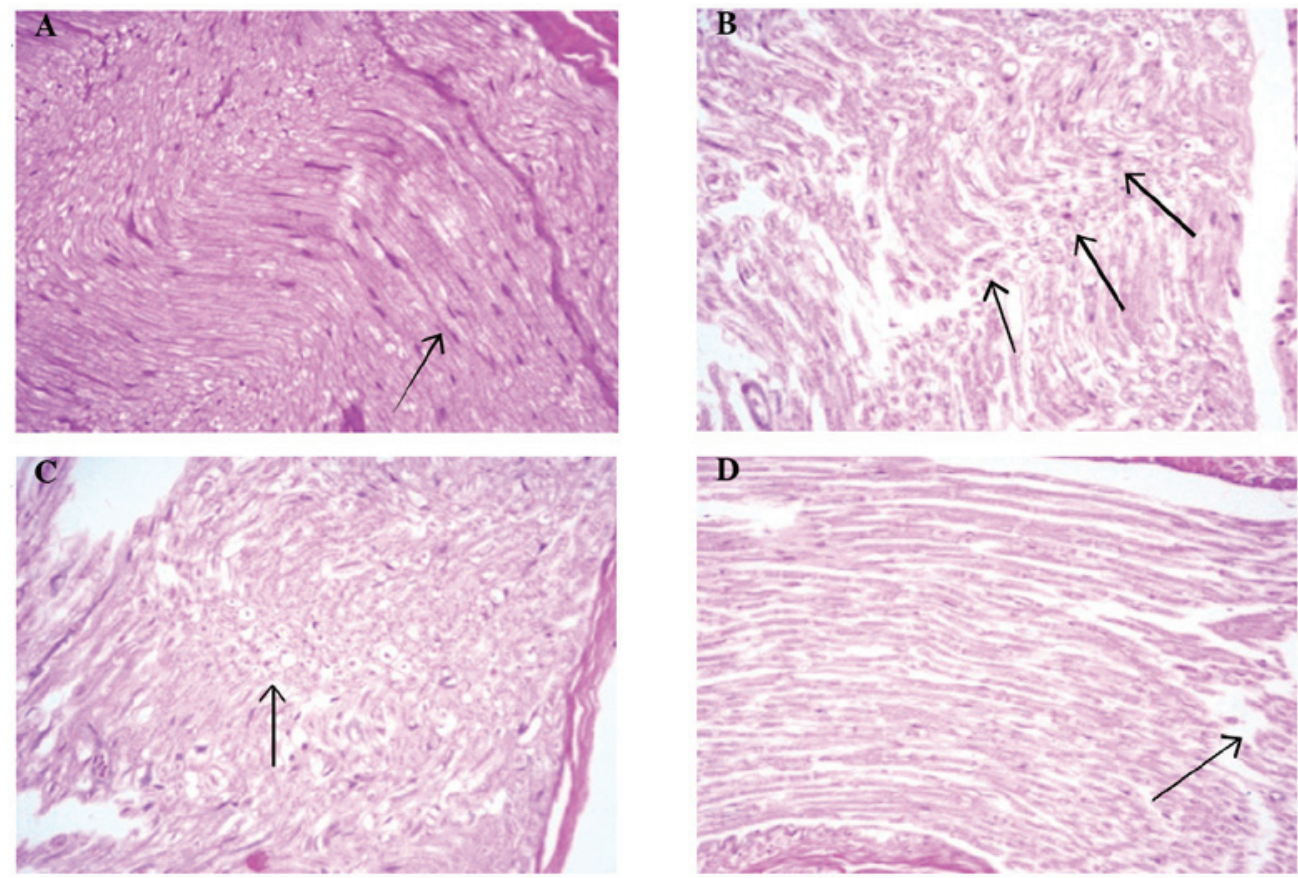

Figure 8. Effects of Gymnema sylvestre (GS) leaf extract on the hematoxylin and eosin histopathological sections (magnification, x200) of sciatic nerves in diabetic rats. Arrows indicate (A) near normal nerve tissue with no degenerative or inflammatory changes in control non-diabetic rats; (B) axonal and lipid degenerated axons with mild focal peripheral axonal loss in diabetic untreated rats; (C) lipoid degeneration of the axons and mild focal peripheral axonal loss in GS50 group; and (D) minimal axonal degenerative changes without a regenerative feature in the GS100 group. 
Furthermore, western blot analysis of NGF protein expression in the sciatic nerve tissue samples revealed a significant downregulation of NGF levels in the diabetic rats when compared with the control group. However, NGF levels were significantly increased in the GS50 and GS100 group rats $(\mathrm{P}<0.05$ and $\mathrm{P}<0.01$, respectively), as compared with the untreated diabetic rats (Fig. 7).

Effects of Gs on the histological features of sciatic nerve in diabetic rats. Histological analyses of sciatic nerve sections from the control group rats revealed near-normal evenly distributed sciatic nerve axons within the myelin sheath, with no indication of degeneration, inflammatory infiltration or other abnormalities (Fig. 8A). Histological sections from the diabetic group rats exhibited focal peripheral axonal loss and lipoid degeneration of the axons, with clusters of regenerative thin myelin axons and few degenerated bodies. Thus, the histological diagnosis was defined as mild degeneration and regenerative neuropathy (Fig. 8B). Sections from the GS50 group rats (Fig. 8C) presented partial focal peripheral axonal loss and regenerating thin myelinated axons. Similarly to the untreated diabetic rats, the histological diagnosis was mild degenerative and regenerative neuropathy. However, histological analysis of the sections from the GS100 group rats exhibited minimal axonal degeneration, with no regenerative features. The histological diagnosis was subsequently indicated as minor degenerative neuropathy (Fig. 8D).

\section{Discussion}

The results of the present study indicate that Gs exerts an ameliorative effect against experimentally induced DN in Wistar rats. Gs was observed to induce an attenuation in the diabetes-induced biochemical and histopathological alterations in the sciatic nerves of rats. Thus, it was hypothesized that this alleviation was a result of the capacity of Gs leaf extract to inhibit STZ-induced hyperglycemia and reduce oxidative and inflammatory injury of the peripheral nervous system.

$\mathrm{DN}$ and associated neuropathic pain are among the most common complications of diabetes, occurring in $\sim 50 \%$ of diabetic patients. In the present study, diabetic rats exhibited a significant delay in paw and tail withdrawal latencies when compared with the control rats. These observations indicated that the diabetic rats had developed mechanical and thermal hyperalgesia, which was consistent with the observations of previous studies $(24,25)$.

In the current study, a significant reduction in serum glucose levels and an increase in insulin levels were observed in the diabetic rats treated with Gs leaf extract $(100 \mathrm{mg} / \mathrm{kg})$ for a period of five weeks, as compared with the untreated diabetic rats. This result is consistent with previous studies that have indicated that Gs may possess antidiabetic properties $(26,27)$. Numerous mechanisms have been proposed to explain the apparent antidiabetic activity of Gs extract. Yoshioka reported that gymnemic acid, the primary active constituent of Gs leaf extract, may be responsible for inhibiting $\mathrm{Na}^{+}$-dependent active glucose transport in the small intestine (28). An additional study suggested that the levels of gastric inhibitory peptide may be suppressed by Gs, producing an antidiabetic effect (29). Furthermore, Sahu et al (30) reported that gymnemic acid blocks the receptor in the intestinal absorptive external layers, similar to oral taste buds, thereby preventing glucose absorption.

Oxidative stress serves a vital function in the pathogenesis of DN $(9,31,32)$ and is known to be induced by hyperglycemia following the autoxidation of monosaccharides and proteins (33). In the present study, significant increases in oxidative stress and LPO biomarkers were observed in the sciatic nerve tissue of diabetic rats. This finding is consistent with previous studies (11), which reported that hyperglycemia resulted in significantly elevated levels of TBARS and reduced levels of GSH in the sciatic nerve. Hyperglycemia is known to reduce the activity of antioxidative enzymes in diabetic model animals, which may involve non-enzymatic glycosylation (34). Similarly, the present study demonstrated an association between DN and oxidative stress through the inhibition of antioxidant enzymes in the sciatic nerve. Thus, neural cells in the sciatic nerve may be more vulnerable to damage as a result of hyperglycemia-induced oxidative stress. Gs treatment of diabetic animals has been observed to protect the peripheral neuronal cells from oxidative damage (35). Furthermore, Gs exhibited antioxidative and LPO-inhibiting properties, as the levels of endogenous antioxidant molecules and the activities of antioxidant enzymes were significantly improved in the sciatic nerves of the diabetic rats. The antidiabetic properties of Gs leaf extract observed in the present study and previous studies may be associated with its strong antioxidative properties (36). Furthermore, these antioxidant and anti-LPO properties have been observed in a number of previous in vivo and in vitro studies (37-39). Gs contains a number of bioactive compounds, including oleanane-type triterpenoid saponins (known as gymnemic acids) $(13,17)$, alkaloids, acidic glycosides and anthroquinones and their derivatives (18). Thus, these groups of molecules may be responsible for the apparent antidiabetic and antioxidative properties exhibited by Gs extract. Previous phytochemical analysis further confirmed the presence of these compounds in the Gs extract (19).

Furthermore, the present study demonstrated an association between the development of DN and elevated levels of proinflammatory mediators, with proinflammatory cytokines detected in the serum and sciatic nerves of the diabetic rats. Increased levels of inflammatory molecules are considered to be produced as a result of hyperglycemia and insulin resistance in diabetes $(40,41)$. In addition, the diabetes-associated overexpression of inflammatory biomarkers is known to provoke neural cell dysfunction and death $(42,43)$. Previous studies have indicated that Gs extract may exert a protective effect against inflammation $(44,45)$. In the present study, treatment of diabetic rats with Gs extract attenuated the elevated levels of cytokines in the serum and sciatic tissues; however, these effects were more marked in the GS100 group rats. Recently, Yasukawa et al (46) observed that treatment with Gs extract suppressed inflammatory tumor promotion in a mouse model of carcinogenesis. Thus, the anti-inflammatory properties of Gs may ameliorate oxidative stress by blocking the nuclear factor (NF)-кB pathway, which subsequently downregulates downstream target genes of NF- $\mathrm{BB}$, such as inducible nitric oxide synthase (iNOS). iNOS catalyzes the oxidative stress-mediated production of NO, which is a vital inflammatory mediator in the pathogenesis of DN. In the present study, 
treatment with Gs extract produced a marked reduction in the STZ-induced elevation of NO levels in the sciatic tissue. This reduction indicates that Gs may inhibit iNOS activity or the expression downstream of NF- $\kappa \mathrm{B}$. However, Gs may also have an inhibitory effect on upstream NF- $\kappa \mathrm{B}$.

Neuronal growth factors, such as NGF and IGF, serve a crucial function in the survival and maintenance of sympathetic and sensory nerves. IGF is a neurological growth factor with a structure similar to that of insulin, and exhibits anabolic effects that inhibit neural cell apoptosis. Furthermore, IGF regulates DNA synthesis and thus the growth and proliferation of nerve cells (47). NGF performs a vital neuroprotective role with the ability to potentiate axonal growth. Pathological conditions that alter NGF expression may result in neuronal dysfunction and death (48). In the present study, levels of IGF and NGF were markedly reduced in the sciatic nerves of the diabetic rats, indicating a loss of neural integrity and an increased rate of nerve cell apoptosis. However, the reduced sciatic tissue levels of IGF and NGF in the diabetic rats were corrected following treatment with Gs. Additionally, histological features of sciatic nerve sections from the Gs-treated groups presented a reduction in degenerated bodies and a preservation of sciatic nerve architecture, as compared with the untreated diabetic rats.

In conclusion, the results of the present study demonstrate the ameliorative properties of Gs extract against chemically-induced DN in rats via its antihyperglycemic, antioxidative and anti-inflammatory properties. Furthermore, morphological examinations indicate that the damage caused to the sciatic nerve by STZ was markedly reduced following the administration of Gs. Therefore, Gs extract application may be useful for the treatment of neuropathy in patients with chronic diabetes.

\section{Acknowledgements}

The authors thank the Deanship of Scientific Research at King Saud University for funding the study through the research group project no. RGP-VPP-266.

\section{References}

1. Callaghan BC, Little AA, Feldman EL and Hughes RA: Enhanced glucose control for preventing and treating diabetic neuropathy. Cochrane Database Syst Rev 6: CD007543, 2012.

2. Galer BS, Gianas A and Jensen MP: Painful diabetic polyneuropathy: Epidemiology, pain description, and quality of life. Diabetes Res Clin Pract 47: 123-128, 2000.

3. Cameron NE and Cotter MA: Effects of antioxidants on nerve and vascular dysfunction in experimental diabetes. Diabetes Res Clin Pract 45: 137-146, 1999.

4. Stevens MJ, Obrosova I, Cao X, Van Huysen C and Greene DA: Effects of DL-alpha-lipoic acid on peripheral nerve conduction, blood flow, energy metabolism, and oxidative stress in experimental diabetic neuropathy. Diabetes 49: 1006-1015, 2000.

5. Calcutt NA: Experimental models of painful diabetic neuropathy. J Neurol Sci 220: 137-139, 2004.

6. Sima AA, Zhang W, Xu G, et al: A comparison of diabetic polyneuropathy in type II diabetic BBZDR/Wor rats and in type I diabetic BB/Wor rats. Diabetologia 43: 786-793, 2000.

7. Edwards JL, Vincent AM, Cheng HT and Feldman EL: Diabetic neuropathy: Mechanisms to management. Pharmacol Ther 120: $1-34,2008$

8. Ceriello A: Controlling oxidative stress as a novel molecular approach to protecting the vascular wall in diabetes. Curr Opin Lipidol 17: 510-518, 2006.
9. Figueroa-Romero C, Sadidi M and Feldman EL: Mechanisms of disease: The oxidative stress theory of diabetic neuropathy. Rev Endocr Metab Disord 9: 301-314, 2008.

10. Zherebitskaya E, Akude E, Smith DR and Fernyhough P: Development of selective axonopathy in adult sensory neurons isolated from diabetic rats: Role of glucose-induced oxidative stress. Diabetes 58: 1356-1364, 2009.

11. Cunha JM, Jolivalt CG, Ramos KM, Gregory JA, Calcutt NA and Mizisin AP: Elevated lipid peroxidation and DNA oxidation in nerve from diabetic rats: Effects of aldose reductase inhibition, insulin, and neurotrophic factors. Metabolism 57: 873-881, 2008.

12. Yeh GY, Eisenberg DM, Kaptchuk TJ and Phillips RS: Systematic review of herbs and dietary supplements for glycemic control in diabetes. Diabetes Care 26: 1277-1294, 2003.

13. Kanetkar P, Singhal R and Kamat M: Gymnema sylvestre: A Memoir. J Clin Biochem Nutr 41: 77-81, 2007.

14. Daisy P, Eliza J and Mohamed Farook KA: A novel dihydroxy gymnemic triacetate isolated from Gymnema sylvestre possessing normoglycemic and hypolipidemic activity on STZ-induced diabetic rats. J Ethnopharmacol 126: 339-344, 2009.

15. RamkumarKM,PonmanickamP, Velayuthaprabhu S,Archunan G and Rajaguru P: Protective effect of Gymnema montanum against renal damage in experimental diabetic rats. Food Chem Toxicol 47: 2516-2521, 2009.

16. Grover JK, Yadav S and Vats V: Medicinal plants of India with anti-diabetic potential. J Ethnopharmacol 81: 81-100, 2002.

17. Ye WC, Zhang QW, Liu X, Che CT and Zhao SX: Oleanane saponins from Gymnema sylvestre. Phytochemistry 53: 893-899, 2000.

18. Surveswaran S, Cai YZ, Xing J, Corke H and Sun M: Antioxidant properties and principal phenolic phytochemicals of Indian medicinal plants from Asclepiadoideae and Periplocoideae. Nat Prod Res 24: 206-221, 2010.

19. Al-Rejaie SS, Abuohashish HM, Ahmed MM, Aleisa AM and Alkhamees O: Possible biochemical effects following inhibition of ethanol-induced gastric mucosa damage by Gymnema sylvestre in male Wistar albino rats. Pharm Biol 50: 1542-1550, 2012.

20. Aleisa AM, Al-Rejaie SS, Abuohashish HM, Ola MS, Parmar MY and Ahmed MM: Pretreatment of Gymnema sylvestre revealed the protection against acetic acid-induced ulcerative colitis in rats. BMC Complement Altern Med 14: 49, 2014.

21. Sedlak J and Lindsay RH: Estimation of total, protein-bound, and nonprotein sulfhydryl groups in tissue with Ellman's reagent. Anal Biochem 25: 192-205, 1968.

22. Kono Y: Generation of superoxide radical during autoxidation of hydroxylamine and an assay for superoxide dismutase. Arch Biochem Biophys 186: 189-195, 1978.

23. Aebi H: Catalase In: Methods in Enzymatic Analysis. Bergmeyer (ed.), New York, pp674-684, 1974.

24. Kamboj SS, Vasishta RK and Sandhir R: N-acetylcysteine inhibits hyperglycemia-induced oxidative stress and apoptosis markers in diabetic neuropathy. J Neurochem 112: 77-91, 2010.

25. AlSharari SD, Al-Rejaie SS, Abuohashish HM, Aleisa AM, Parmar MY and Ahmed MM: Ameliorative potential of morin in streptozotocin-induced neuropathic pain in rats. Trop J Pharm Res 13: 1429-1436, 2014.

26. El Shafey AAM, El-Ezabi MM, Seliem MME, Ouda HHM and Ibrahim DS: Effect of Gymnema sylvestre R. Br. leaves extract on certain physiological parameters of diabetic rats. Journal of King Saud University - Science 25: 135-141, 2013.

27. Verma N, Shakya VK and Saxena RC: Antidiabetic activity of glycoside isolated from Gymnema sylvestre in streptozotocin induced diabetic rats. Asian J Chem 20: 5033-5036, 2008.

28. Yoshioka S: Inhibitory effects of gymnemic acid and an extract from the leaves of Ziziphus jujuba on glucose absorption in the rat small intestine. J Yonago Med Assoc 37: 142-154, 1986.

29. Fushiki T, Kojima A, Imoto T, Inoue K and Sugimoto E: An extract of Gymnema sylvestre leaves and purified gymnemic acid inhibits glucose-stimulated gastric inhibitory peptide secretion in rats. J Nutr 122: 2367-2373, 1992.

30. Sahu NP, Mahato SB, Sarkar SK and Poddar G: Triterpenoid saponins from Gymnema sylvestre. Phytochemistry 41: 1181-1185, 1996.

31. Bolajoko EB, Mossanda KS, Adeniyi F, Akinosun O, Fasanmade A and Moropane M: Antioxidant and oxidative stress status in type 2 diabetes and diabetic foot ulcer. S Afr Med J 98: 614-617, 2008. 
32. Julius U, Drel VR, Grässler J and Obrosova IG: Nitrosylated proteins in monocytes as a new marker of oxidative-nitrosative stress in diabetic subjects with macroangiopathy. Exp Clin Endocrinol Diabetes 117: 72-77, 2009.

33. Bonnefont-Rousselot D: Glucose and reactive oxygen species. Curr Opin Clin Nutr Metab Care 5: 561-568, 2002.

34. Halliwell B: Drug antioxidant effects. A basis for drug selection? Drugs 42: 569-605, 1991

35. Preuss HG, Jarrell ST, Scheckenbach R, Lieberman S and Anderson RA: Comparative effects of chromium, vanadium and Gymnema sylvestre on sugar-induced blood pressure elevations in SHR. J Am Coll Nutr 17: 116-123, 1998.

36. Kang MH, Lee MS, Choi MK, Min KS and Shibamoto T: Hypoglycemic activity of Gymnema sylvestre extracts on oxidative stress and antioxidant status in diabetic rats. J Agric Food Chem 60: 2517-2524, 2012.

37. Kumar V, Bhandari U, Tripathi CD and Khanna G: Evaluation of antiobesity and cardioprotective effect of Gymnema sylvestre extract in murine model. Indian J Pharmacol 44: 607-613, 2012.

38. Sharma K, Singh U, Vats S, Priyadarsini K, Bhatia A and Kamal R: Evaluation of evidenced-based radioprotective efficacy of Gymnema sylvestre leaves in mice brain. J Environ Pathol Toxicol Oncol 28: 311-323, 2009.

39. Rachh PR, Patel SR, Hirpara HV, et al: In vitro evaluation of antioxidant activity of Gymnema sylvestre R. BR. leaf extract. Rom J Biol 54: 141-148, 2009.

40. Brownlee M: The pathobiology of diabetic complications: A unifying mechanism. Diabetes 54: 1615-1625, 2005.

41. Navarro-González JF and Mora-Fernández C: The role of inflammatory cytokines in diabetic nephropathy. J Am Soc Nephrol 19 433-442, 2008.
42. Li J, Wei GH, Huang H, Lan YP, Liu B, Liu H, Zhang W and Zuo YX: Nerve injury-related autoimmunity activation leads to chronic inflammation and chronic neuropathic pain. Anesthesiology 118: 416-429, 2013.

43. Djordjevic A, Bursać B, Veličković N, Vasiljević A and Matić G: The impact of different fructose loads on insulin sensitivity, inflammation, and PSA-NCAM-mediated plasticity in the hippocampus of fructose-fed male rats. Nutr Neurosci 18: 66-75, 2015.

44. Diwan PV, Margaret I and Ramakrishna S: Influence of Gymnema sylvestre on inflammation. Inflammopharmacology 3: 271-277, 1995.

45. Jitender KM, Manvi F, Alagawadi K and Noolvi M: Evaluation of anti-inflammatory activity of Gymnema sylvestre leaves extract in rats. IJGP 2: 114-115, 2008.

46. Yasukawa K, Okuda S and Nobushi Y: Inhibitory effects of Gymnema (Gymnema sylvestre) leaves on tumour promotion in two-stage mouse skin carcinogenesis. Evid Based Complement Alternat Med 2014: 328684, 2014.

47. Scarth JP: Modulation of the growth hormone-insulin-like growth factor (GH-IGF) axis by pharmaceutical, nutraceutical and environmental xenobiotics: An emerging role for xenobiotic-metabolizing enzymes and the transcription factors regulating their expression. A review. Xenobiotica 36: 119-218, 2006.

48. Freeman RS, Burch RL, Crowder RJ, Lomb DJ, Schoell MC, Straub JA and Xie L: NGF deprivation-induced gene expression: After ten years, where do we stand? Prog Brain Res 146: 111-126, 2004. 